\title{
Involved microRNAs in alternative polyadenylation intervene in breast cancer via regulation of cleavage factor "CFIm25"
}

\author{
Mona Tamaddon ${ }^{1}$, Gelareh Shokri ${ }^{1}$, Seyed Mohammad Ali Hosseini Rad ${ }^{2}$, Iman Rad ${ }^{1}$, \\ Àmirnader Emami Razavi ${ }^{3}$ \& Fatemeh Kouhkan ${ }^{1 \times}$
}

Cleavage factor "CFIm25", as a key repressor at proximal poly (A) site, negatively correlates to cell proliferation and tumorigenicity in various cancers. Hence, understanding CFIm 25 mechanism of action in breast cancer would be a great benefit. To this aim four steps were designed. First, potential miRNAs that target 3'-UTR of CFIm25 mRNA, retrieved from Targetscan web server. Second, screened miRNAs were profiled in 100 breast cancer and 100 normal adjacent samples. Third, miRNAs that their expression was inversely correlated to the CFIm25, overexpressed in MDA-MB-231 cell line, and their effect on proliferation and migration monitored via MTT and wound healing assays, respectively. Fourth, interaction of miRNAs of interest with 3'-UTR of CFIm25 confirmed via luciferase assay and western blot. Our results indicate that CFIm 25 considerably down-regulates in human breast cancer tissue. qRT-PCR assay, luciferase test, and western blotting confirm that CFIm 25 itself could be directly regulated by oncomiRs such as miR-23, $-24,-27,-135,-182$ and -374 . Besides, according to MTT and wound healing assays of cell lines, CFIm25 knockdown intensifies cell growth, proliferation and migration. Our results also confirm indirect impact of CFIm25 on regulation of mRNA's 3'-UTR length, which then control corresponding miRNAs' action. miRNAs directly control CFIm 25 expression level, which then tunes expression of the oncogenes and tumor proliferation. Therefore, regulation of CFIm25 expression level via miRNAs is expected to improve treatment responses in breast cancer.

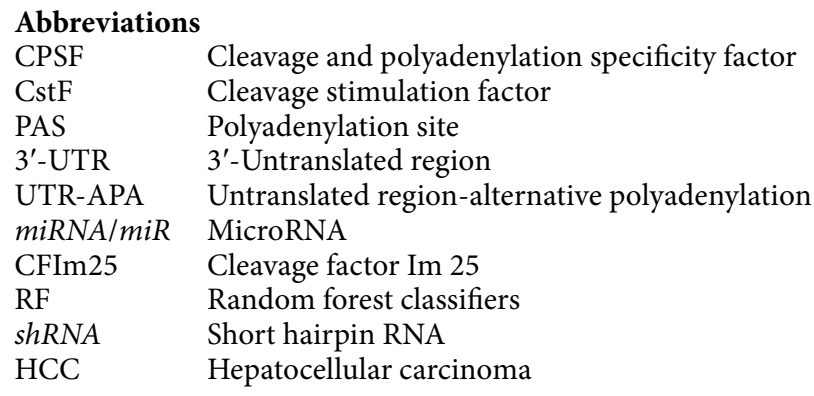

Living organisms apply complicated mechanisms of gene regulation to generate different cell types, which result in various behaviors from a single genome $\mathrm{e}^{1,2}$. Dynamic and highly polymorphic nature of mRNA polyadenylation, as a functional aspect of gene regulation, is one of the most recent discoveries in this field ${ }^{3,4}$. Cleavage and polyadenylation of nascent mRNA accomplishes via application of two large multimeric complexes, which named as cleavage-polyadenylation specificity factor (CPSF) and cleavage stimulation factor (CstF), respectively. The

\footnotetext{
${ }^{1}$ Stem Cell Technology Research Center, No. 9, East 2nd, St., Farhang Blvd., Saadat Abad St., Tehran 1997775555, Iran. ${ }^{2}$ Department of Microbiology and Immunology, University of Otago, Dunedin, Otago 9010, New Zealand. ${ }^{3}$ Iran National Tumor Bank, Cancer Biology Research Center, Cancer Institute of Iran, Tehran University of Medical Sciences, Tehran, Iran. ${ }^{\circledR}$ email: f.kouhkan@yahoo.com
} 
CPSF recognizes polyadenylation site (PAS) element located $\sim 19$ nucleotides away from the polyA site in the 3'-UTR of mRNAs, while CstF directly interacts with CPSF and binds to GU-rich element at downstream of cleavage site ${ }^{5-7}$. Most human genes harbor multiple polyadenylation signals, which results in variable $3^{\prime}$-UTR length without changing the coding region, or produce different protein isoforms through the usage of poly(A) sites residing in internal introns/exons ${ }^{3,8-10}$.

The UTR-alternative polyadenylation ${ }^{1}$ site play critical roles in various biological networks through changes that occur in 3'-UTR length. For example, UTR-APA could influence the stability, translation efficiency and subcellular localization of mRNA. The UTR-APA also regulates protein transport by altering interaction of RNA binding proteins with the $3^{\prime}$-UTR. Therefore, UTR-APA site quantitatively affects corresponding protein expression through a complicated mechanism ${ }^{10,11}$. UTR-APA site controls length of the $3^{\prime}$-UTR, which then controls miRNAs' binding sites availability at the $3^{\prime}$-UTR. The choice of poly (A) site can be influenced by a key factor in APA, which is human cleavage factor $\operatorname{Im}(\mathrm{CFIm})^{12}$.

The CFIm is an essential component in the pre-mRNA 3'-UTR processing complex. It contains three polypeptides of 25, 59 and $68 \mathrm{kDa}$ that are designated as CFIm25 (CPSF5/NUDT21), CFIm59 (CPSF7, cleavage and polyadenylation specificity factor 7), and CFIm68 (or CPSF6). Diminution of CFIm25 level induces a global recruitment of weak polyA, placed completely proximal to the stop codon, while enhances cell proliferation. Previous studies confirmed down-regulation of CFIm 25 in tumor tissue compared to the normal tissue. Besides, diminution of CFIm25 level comes up with shorter length of $3^{\prime}$-UTR in various oncogenes and induces proliferation of tumor cells ${ }^{13-15}$. However, mechanism that regulates CFIm25 expression, which then correlates to the occurrence and development of cancer, remains unknown.

miRNAs are tiny non-coding RNAs that are involved in post-transcriptional gene regulation. They target cognate mRNAs contain complementary sequences in their 3'-UTR, which either prevents mRNA translation or induce its degradation ${ }^{16-18}$. Numerous miRNAs have been shown to be aberrantly expressed, and act as oncogenes in several types of cancer such as breast cancer ${ }^{19-23}$. Besides, miRNAs mimic tumor suppressor roles in many cases. That's why loss of miRNA expression correlates with inception or the aggressiveness of tumors ${ }^{24,25}$.

In this study, we checked if miRNAs are involved with regulation of CFIm 25 expression. To examine regulatory impact of miRNAs on endogenously APA master regulator CFIm25, correlation of oncogenic miRNAs with CFIm 25 investigated in clinical samples of breast cancer patients. Then, we explored impact of transfected miRNAs in breast cancer cell line, in a set of parallel separate experiments. Identification of numerous oncomiRs that suppress CFIm25, which then induce proximal polyadenylation and mediate in production of shortened oncogenes in breast cancer, confirms involvement of miRNAs in regulation of CFIm 25.

\section{Results}

Correlation of CFIm 25 expression level with oncogenic miRNAs in breast cancer clinical samples and cell lines. According to bioinformatics analysis, $m i R-23, m i R-24, m i R-27, m i R-96, m i R-135$, $m i R-182, m i R-221, m i R-222$ and $m i R-374$ found to target CFIm 25 beside their oncogenic potential. In order to determine whether CFIm 25 expression is controlled by miRNAs of interest in breast cancer, we measured the expression levels of potentially involved miRNAs in breast cancer patients $(\mathrm{n}=100)$ and normal adjacent tissues $(\mathrm{n}=100)$ using $m i R$-quantitative qRT-PCR analysis. Results showed considerable elevated level of miRNAs in samples acquired from patients (at least in 6 out of 9 monitored miRNAs), while CFIm 25 expression level was diminished significantly (Fig. 1a, b). In the next step, patients categorized as low or high CFIm25-expressing tumors, using the median expression for CFIm 25 in the normal sample as a cut-off.

Since CFIm 25 level differs in samples acquired from patients, their correspondingly correlated miRNAs might be variable too. By this regard, distribution of the median expression of nine miRNAs of interest evaluated respect to both low and high levels of CFIm25 expression (Fig. 1c).

The median gene expression level of $m i R-23, m i R-24, m i R-27, m i R-135, m i R-182, m i R-221$ and $m i R-374$ was significantly higher in the patients expressing lower levels of CFIm25, compared to those expressing higher levels $(p<0.05)$.

Intervention of CFIm25 in length determination of proto-oncogenes' $3^{\prime}$-UTR. Three out of five proto-oncogenes interact efficiently with CFIm 25 and CPSF1 either at proximal or distal of $3^{\prime}$-UTR while the other two proto-oncogenes (EGFR: NM_005228.5, AKT3: NM_005465.5) interact with both proteins merely at distal of the $3^{\prime}$-UTR (Table 1). Therefore, exclusive interaction of CFIm25/CPSF1 complex with distal region of EGFR and AKT3 3'-UTR coding mRNA, would guarantee suppression of breast cancer progression.

Some of the potentially involved miRNAs in breast cancer, down-regulate CFIm25 in MDA-MB-231 cell line. To confirm the impact of miRNAs on CFIm25 regulation in clinical samples, MDAMB-231 stable cell lines were generated using miRNA containing lentivirus transduction. We determined that enforced expressions of $m i R-23, m i R-24, m i R-27, m i R-135, m i R-182$ and $m i R-374$ significantly down-regulated CFIm25 $(p<0.05)$, while made no change in cells that were transducted with Ctrl-vector, miR-96, miR-221 and $m i R-222$ (Fig. 2a). Up-regulation of $m i R-23$ and $m i R-374$ down-regulated CFIm 25 the most, compared to other miRNAs including $m i R-24, m i R-27, m i R-135$ and $m i R-182$ (Fig. 2a). According to image analysis of western blot gels via Image J software, CFIm 25 protein level notably decreased while $m i R-23,27$, and 374 were up-regulated (Fig. 2b).

In the next step, we investigated whether $3^{\prime}$-UTR of CFIm 25 has functional targets for predicted miRNAs. To this aim, full-length sequence of CFIm25 3'-UTR cloned to psiCHECK-2 vector, which is placed at downstream of Renilla luciferase reporter gene (CFIm25-psiCHECK2). In a parallel experiment, as negative controls, the seed sequence of $m i R-23, m i R-24, m i R-27, m i R-135, m i R-182$ and $m i R-374$ mutated intentionally at certain points. The 
(A)

(B)
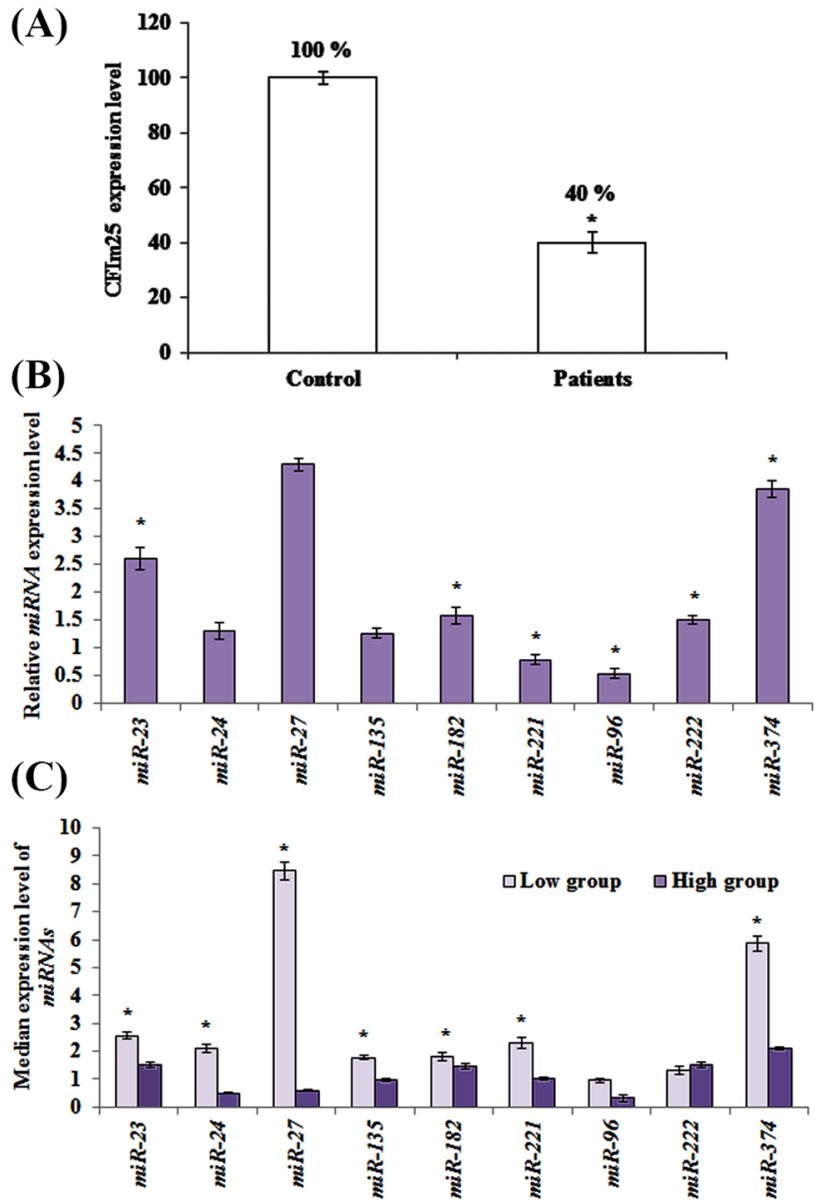

Figure 1. Comparative expression of CFIm 25 vs involved miRNAs. (a) CFIm25 expression level in samples acquired from breast cancer patients and control group. CFIm 25 is significantly lower in patients compared to controls. (b) Relative gene expression of miRNAs in patients. (c) Distribution of miRNA expression in patients according to low and high expression levels of CFIm25. Patients categorized as low or high CFIm25-expressing tumors, using median expression of CFIm 25 in normal samples as a cut-off. Data represent mean of at least three independent experiments. The star above the bars, represents significant difference respect to the corresponding control reference. The results are relative gene expression after normalization with $\beta$-actin for CFIm 25 and SNORD47 for miRNA genes, using $2^{-\Delta \Delta C t}$ method.

stem-loop structures that formed following introduction of mutations, cloned to $p C D H$-TurboGFP vector (MutmiRNAs) in shRNA format. The HEK293 cells transiently transfected with the CFIm25-pSICHECK2 construct and WT-miRNAs, which led to a significant decrease of reporter activity compared to the control (Fig. 2c). Activity of the reporter construct was unaffected by a simultaneous transfection with Mut-miRNAs carried a mutated seed sequence. Taken together, these data strongly suggest that $m i R-23, m i R-24, m i R-27, m i R-135, m i R-182$ and miR-374 directly bind to 3'-UTR of CFIm25's mRNA.

According to our findings that suggested $m i R-23$ and $m i R-347$ had something to do with CFIm 25 downregulation, we used $m i R-O f f-23$ and $m i R-O f f-374$ to silence $m i R-23$ and $m i R-374$. Now, by using $m i R-O f f-23$ and $m i R-O f f-374$, we can confirm if silencing of $m i R-23$ and $m i R-374$ directly controls overexpression of CFIm 25 in breast cancer's cell line or not. To this aim, miR-Off-23 and miR-Off-374 transducted to MDA-MB-231 cell line and efficiency of transduction monitored using qRT-PCR analysis. Transduction of $m i R-O f f-23$ and miR-Off-374 resulted in significant overexpression of CFIm 25 as demonstrated in Fig. $2 \mathrm{~d}$ (by 3.3 and 2.89 fold increase in case of $m i R-O f f-23$ and $m i R-O f f-374$, respectively).

Selected miRNAs can regulate cell proliferation in breast cancer cell line partly by modulating CFIm25 mRNA. To determine importance of CFIm 25 in regulation of MDA-MB-231 proliferation, we intentionally performed MTT and wound healing assays in miRNAs transduced MDA-MB-231 cells. Result of the MTT assay confirmed increment of "cell proliferation index" in transduced cells as compared with that of the control groups $(p<0.05$; Fig. 3a).

To clarify direct impact of miRNAs on cell proliferation through regulation of 3'-UTR of CFIm25 mRNA, MDA-MB-231 cells once transduced with $m i R-O f f-23 / m i R-O f f-374$ and assessed by MTT assay. In the second approach, transduced cells transfected by shCFIm 25 vector and evaluated by MTT assay. When miR-Off-23 and 


\begin{tabular}{|c|c|c|c|c|c|c|c|c|}
\hline \multirow[b]{2}{*}{$\begin{array}{l}\text { 3' UTR of the } \\
\text { mRNA }\end{array}$} & \multirow{2}{*}{$\begin{array}{l}\text { Possible } \\
\text { CFIm } \\
\text { interaction } \\
\text { site } \\
\text { (TGTA) }\end{array}$} & \multirow{2}{*}{ 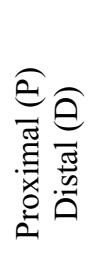 } & \multirow{2}{*}{ 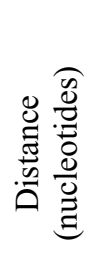 } & \multicolumn{2}{|c|}{$\begin{array}{c}\text { Poly-adenylation signal } \\
\text { (PAS) }\end{array}$} & \multirow{2}{*}{ 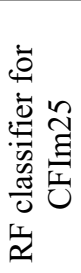 } & \multirow{2}{*}{ 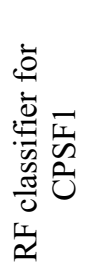 } & \multirow{2}{*}{ 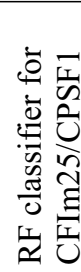 } \\
\hline & & & & ATTAAA & AATAAA & & & \\
\hline \multirow{8}{*}{$\begin{array}{c}\text { EGFR: } \\
\text { NM_005228.5 }\end{array}$} & \multirow{3}{*}{$1482-1487$} & $\mathrm{P}$ & 208 & - & $1695-1700$ & 0.45 & 0.55 & 0.7 \\
\hline & & \multirow{7}{*}{$\mathrm{D}$} & 300 & - & $1787-1793$ & 0.45 & 0.7 & 0.7 \\
\hline & & & 985 & - & $2445-2450$ & 0.45 & 0.6 & 0.65 \\
\hline & $2384-2387$ & & 19 & $2406-2411$ & - & 0.45 & 0.75 & 0.7 \\
\hline & $3429-3434$ & & 223 & - & $3657-3662$ & 0.15 & 0.75 & 0.6 \\
\hline & $4554-4559$ & & 71 & $4828-4833$ & - & 0.5 & 0.75 & 0.7 \\
\hline & $4972-4977$ & & 203 & - & $5180-5185$ & 0.35 & 0.7 & 0.65 \\
\hline & $5856-5859$ & & 195 & $5930-5935$ & - & 0.6 & 0.6 & 0.6 \\
\hline \multirow{7}{*}{$\begin{array}{c}\text { MYC: } \\
\text { NM_002467.6 }\end{array}$} & $263-266$ & \multirow{2}{*}{$\mathrm{P}$} & 11 & $276-282$ & \multirow{2}{*}{-} & 0.5 & 0.5 & 0.5 \\
\hline & $212-215$ & & 62 & $276-282$ & & 0.55 & 0.7 & 0.6 \\
\hline & $263-266$ & \multirow{5}{*}{$\mathrm{D}$} & 29 & - & $295-300$ & 0.5 & 0.65 & 0.45 \\
\hline & $282-285$ & & 151 & - & $436-441$ & 0.4 & 0.6 & 0.55 \\
\hline & $1043-1048$ & & 622 & - & $1670-1675$ & 0.45 & 0.7 & 0.7 \\
\hline & $1802-1805$ & & 167 & - & 1972-1977 & 0.55 & 0.75 & 0.7 \\
\hline & 1934-1937 & & 35 & - & $1972-1977$ & 0.55 & 0.75 & 0.65 \\
\hline \multirow{3}{*}{$\begin{array}{c}\text { PTEN: } \\
\text { AF067844.1 }\end{array}$} & $200-203$ & \multirow{2}{*}{$\mathrm{P}$} & 59 & - & $262-267$ & 0.3 & 0.55 & 0.6 \\
\hline & $213-216$ & & 50 & - & $262-267$ & 0.45 & 0.55 & 0.55 \\
\hline & $710-713$ & $\mathrm{D}$ & 167 & $880-885$ & - & 0.5 & 0.75 & 0.75 \\
\hline \multirow{6}{*}{$\begin{array}{c}\text { AKT2: } \\
\text { NM_001626.6 }\end{array}$} & $1504-1507$ & $\mathrm{P}$ & 11 & - & $1518-1523$ & 0.5 & 0.6 & 0.55 \\
\hline & $2710-2713$ & \multirow{5}{*}{$\mathrm{D}$} & 39 & - & $2752-2757$ & 0.3 & 0.6 & 0.55 \\
\hline & $2710-2713$ & & 54 & - & $2767-2772$ & 0.3 & 0.6 & 0.5 \\
\hline & $2873-2876$ & & 263 & - & 3139-3144 & 0.35 & 0.6 & 0.55 \\
\hline & $2873-2876$ & & 293 & $3169-3174$ & - & 0.35 & 0.7 & 0.55 \\
\hline & $3530-3533$ & & 0 & - & $3533-3538$ & 0.55 & 0.8 & 0.65 \\
\hline \multirow{10}{*}{$\begin{array}{c}\text { AKT3: } \\
\text { NM_005465.5 }\end{array}$} & $1560-1563$ & \multirow[t]{2}{*}{$\mathrm{P}$} & 93 & $1656-1661$ & \multirow{2}{*}{-} & 0.25 & 0.8 & 0.6 \\
\hline & $1611-1614$ & & 42 & & & 0.45 & 0.8 & 0.6 \\
\hline & 3054-3057 & \multirow{8}{*}{$\mathrm{D}$} & 81 & - & $3135-3140$ & 0.5 & 0.55 & 0.6 \\
\hline & $3392-3395$ & & 70 & - & $3462-3467$ & 0.35 & 0.7 & 0.55 \\
\hline & $3392-3395$ & & 137 & $3532-3537$ & - & 0.35 & 0.7 & 0.55 \\
\hline & $4281-4284$ & & 40 & $4324-4329$ & - & 0.5 & 0.7 & 0.6 \\
\hline & $4281-4284$ & & 87 & - & $4371-4376$ & 0.5 & 0.65 & 0.55 \\
\hline & $4749-4752$ & & 214 & - & $4966-4971$ & 0.4 & 0.75 & 0.7 \\
\hline & $4749-4752$ & & 245 & $4997-5002$ & - & 0.4 & 0.8 & 0.75 \\
\hline & $5430-5433$ & & 72 & - & $5505-5510$ & 0.45 & 0.65 & 0.6 \\
\hline
\end{tabular}

Table 1.. Proto-oncogenes that CFIm 25 and CPSF1 target their 3'UTR mRNA. The cases that are highlighted in dark grey represent proto-oncogenes with positive interaction probability. The RF (Random Forest) classifier is a probability index, which falls between 0 (the lowest probability) to 1 (the highest probability).

miR-Off-374 transduced to cells and transiently co-transfected with shCFIm 25 vector (Fig. 3b, c), CFIm 25 expression level diminished and cell proliferation significantly increased (Fig. 3d). Collectively, these results suggest that miR-Off-23/-374 vectors up-regulated CFIm25 protein level, which eventually suppressed proliferation of cancer cells.

Wound healing assay exemplifies CFIm25 balance with miR-23, -374. The wound healing assay designed to evaluate role of miR-Off-23/miR-Off-374 in MDA-MB-231 in cell migration. As what is shown in 
(a)

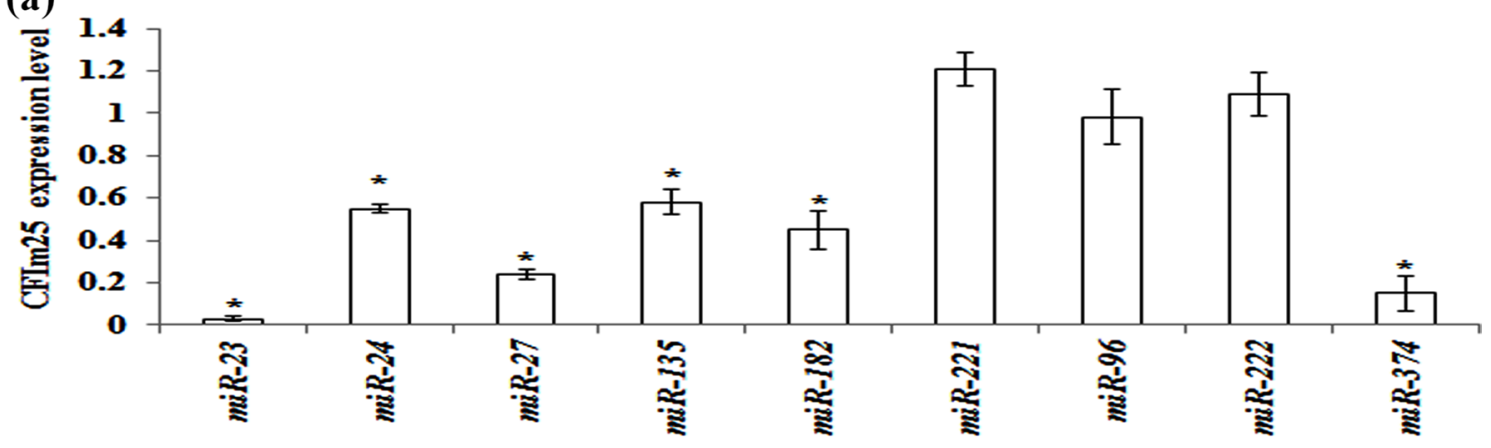

(b)

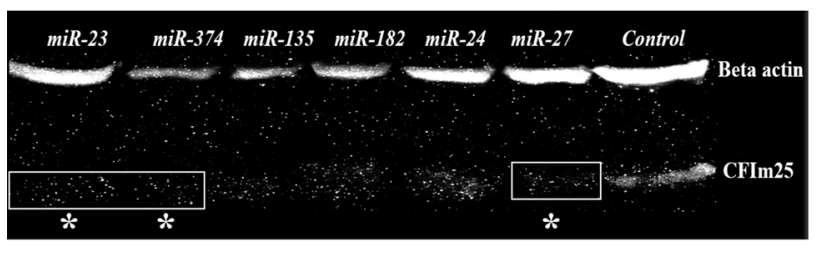

(d)

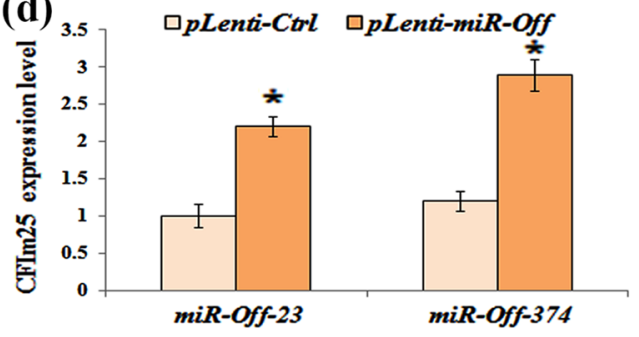

(c)

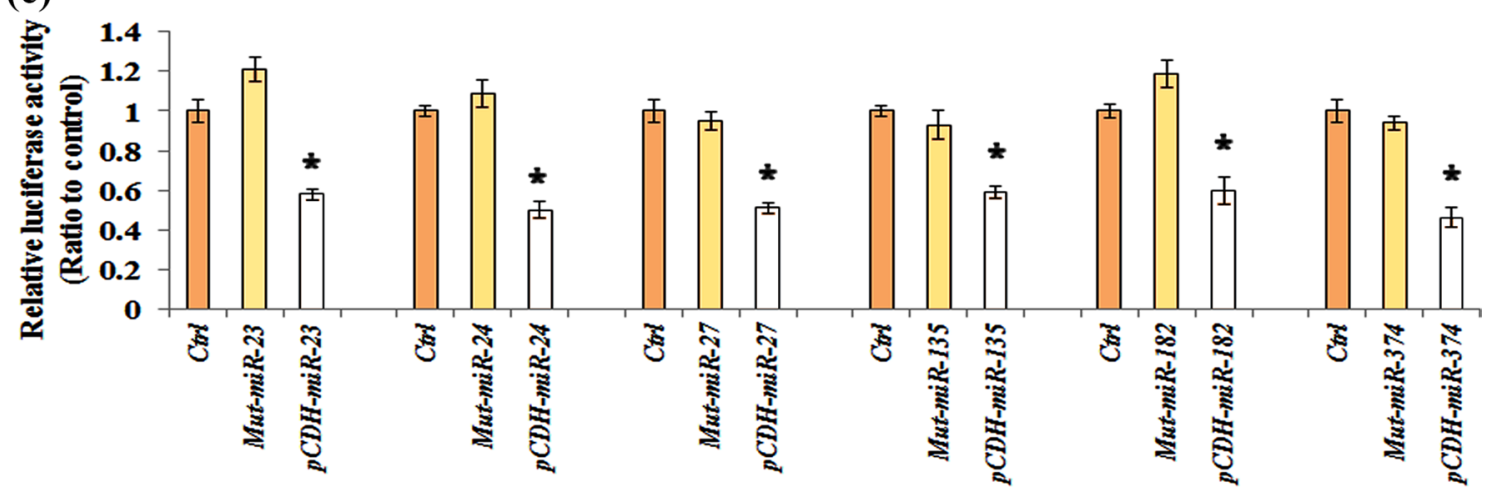

Figure 2. Some of predicted miRNAs directly regulate CFIm 25 expression. (a) CFIm 25 expression level in cell line with enforced expressions of miRNAs of interest, monitored by Real-time PCR and (b) Western blot technique. The western blot bonds cropped from a same gel and membrane. The original image of the membrane and gel could be found in supplementary Figs. 1 and 2. (c) Luciferase assay of the HEK293 cells that are transiently transfected with CFIm25-pSICHECK2 construct and WT-miRNAs/mut-miRNAs. (d) Expression level of CFIm 25 in cells transducted with either of $m i R-O f f-23$ or miR-Off-374 constructs. Protein normalization performed against $\beta$-actin expression. Columns are mean of three different experiments and error bars are standard deviation $(p<0.05)$. The star represents significant difference respect to the corresponding control reference.

Fig. 4, down-regulation of $m i R-23$ and $m i R-374$ (by $m i R$-off-23, -374 transfection) significantly decreased migration ability of transduced cells. However, shCFIm 25 vector improved migration.

Results of migration assay showed that number of migrated cells in the miR-Off-23/miR-Off-374 transduced group, was less than that in the shCFIm 25 group. This last finding confirmed that the migration ability of MDAMB-231 cells was inhibited by miR-Off-23/miR-Off-374, which had been found previously to be under influence of CFIm 25 expression level (Fig. 5).

\section{Discussion}

It is conformed that most of the human genes have multiple polyadenylation signals, which dynamically regulate their variations in $3^{\prime}$-UTR length ${ }^{26}$. The CFIm 25, as a newly discovered repressor of proximal poly (A) site usage, has a significant role in UTR-APA site regulation. The CFIm25, as a subunit of cleavage factor complex, recruits CPSF6 and/or CPSF7 along with CPSF5, and activates 3'-mRNA cleavage site and polyadenylation processing machinery ${ }^{3,11}$. Down-regulation of CFIm25, significantly diminishes "distal poly-A signal" length, and enhances tumorigenic properties and size of the tumor cells. However, CFIm 25 overexpression reduces aforementioned properties and inhibits tumor growth. An explanation for this is that by choosing a proximal PAS, the 3'-UTR length of the mRNAs shortens, which then eliminates miRNAs binding sites and revokes regulation through miRNAs $s^{11,27-29}$. Here, we approved CFIm 25 is indirectly involved in regulation of many genes through changing 

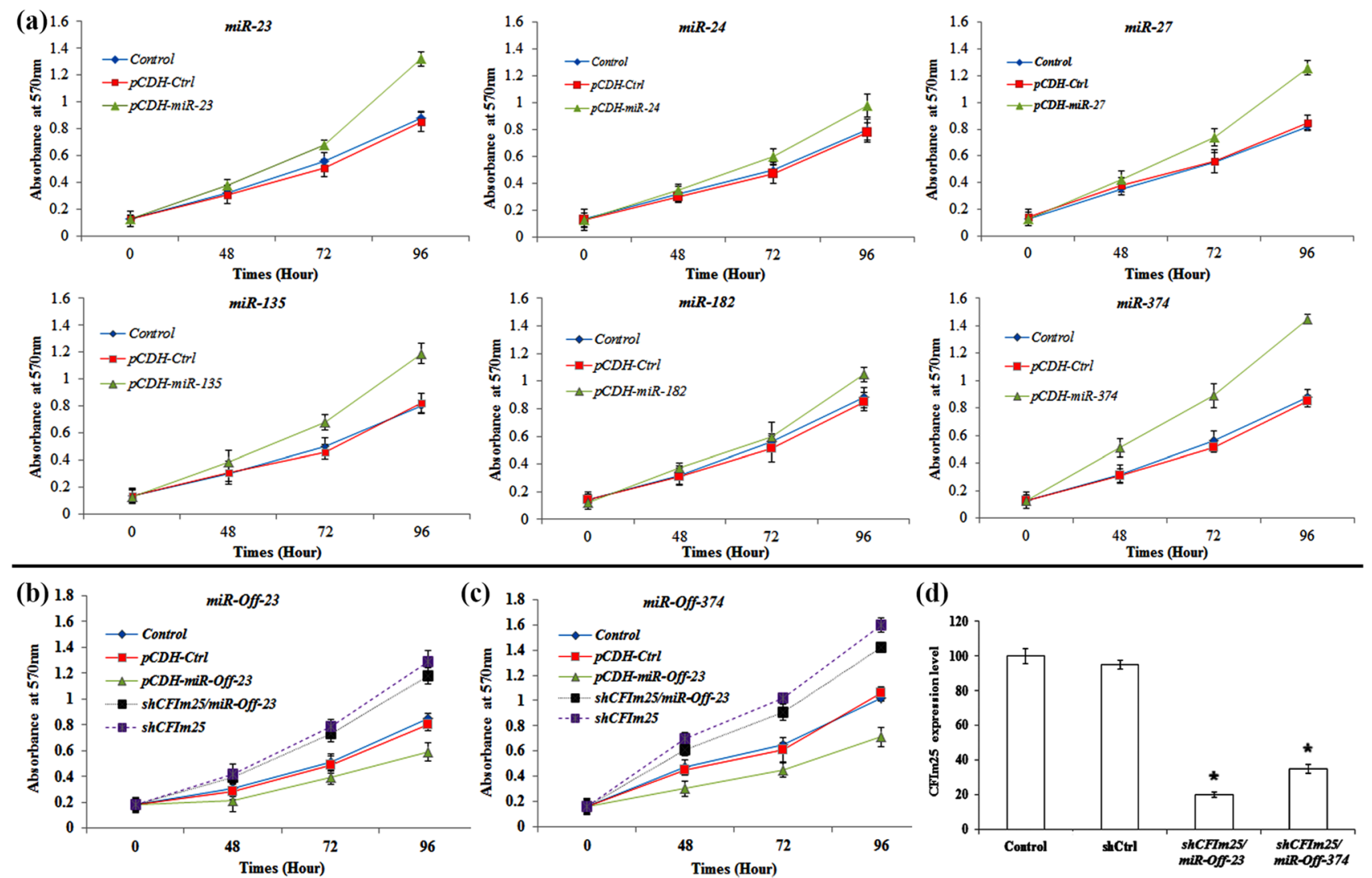

Figure 3. Viability test of MDA-MB-231 cells that were transducted with different cloned miRNAs. (a) MTT assay of MDA-MB-231 cells that are transducted with miRNAs of interest. MTT assay of transducted MDA-MB-231 cells by (b) miR-Off-23 and (c) miR-Off-374 that transfected by shCFIm 25 vector. Each time point was expressed as total absorbance at $570 \mathrm{~nm}$ after background subtraction (Y axis). Points are mean of three experiments and error bars represent standard deviation. (d) CFIm 25 expression level in shCFIm 25 transfected cells. The star above the bar, represents the significant difference respect to the corresponding control reference.

3'-UTR length, which then eliminates binding sites of their corresponding miRNAs. Nonetheless, more studies are still needed to clarify comprehensive regulatory mechanism of CFIm 25 gene by miRNAs.

Although breast cancer is the most common cancer diagnosed in women, survival rates of breast cancer have improved recently, which is mostly due to factors such as a new personalized approach to cancer treatment and a better understanding of the molecular mechanism of the disease ${ }^{30}$. In this study, we used human breast cancer specimens to analyze their CFIm 25 expression profile. We confirmed that CFIm 25 is significantly down-regulated in breast cancer samples, compared to the controls. This phenomenon is consistent with the previous study ${ }^{15}$ that introduced CFIm 25 as APA regulator that was also down-regulated in glioblastoma and came up with cancer proliferation and tumorigenicity ${ }^{16}$. Besides, it was reported that expression level of CFIm 25 in hepatocellular carcinoma (HCC) was negatively correlated to the metastatic potential of HCC cell line through increasing E-cadherin level ${ }^{31}$, while glutaminase $m R N A$ isoforms, which contain distinct 3'-UTR (KGA and GAC), showed a complex interplay between RNA processing and microRNA repression in controlling glutamine metabolism in cancer cells ${ }^{14}$.

In the next step of the current study, we selected nine oncomiRs using bioinformatics analysis. They all confirmed to have recognition sites in the $3^{\prime}$-UTR of the CFIm25 (miR-23: 2 sites, miR-24: 2 sites, miR-27: 3 sites, $m i R-96: 1$ site, $m i R-135: 2$ sites, $m i R-182: 1$ site, $m i R-221: 2$ sites, $m i R-222: 2$ sites and $m i R-374: 4$ sites). So far, several studies investigated role of oncomiRs in different cancers including breast cancer ${ }^{20,24}$. However, no experimental work has performed to clarify effects of these miRNAs on CFIm 25 expression level and, as a result, in regulation of UTR-APA site. When clinical samples divided into two groups, whose CFIm 25 expression was more or lower than the median expression of control samples, a negative correlation between level of $m i R-23$, $m i R-24, m i R-27, m i R-135, m i R-182$ and $m i R-374$ and CFIm25's expression was found. This negative correlation in turn suggests that CFIm 25 is being regulated by aforementioned miRNAs. Besides, in vitro data from transduced MDA-MB-231 cells confirmed that high levels of $m i R-23, m i R-24, m i R-27, m i R-135, m i R-182$ and $m i R-374$ was negatively correlated with either CFIm 25 mRNA or its protein content.

According to previous reports, low level of CFIm 25 leads to cancer proliferation and migration ${ }^{14,28,31}$, which is in agreement with our MTT and wound healing assay results. Importantly, functional regulatory role of miR-23 and miR-374 on CFIm 25 mRNA, approved via MTT assay. This assay confirmed increased growth of MDA-MB-231 cells, which were co-transduced with $m i R-O f f-23 / m i R-O f f-374$ and CFIm 25 knock-down vectors. CFIm 25 is known to be crucial in controlling invasion and metastasis of $\mathrm{HCC}^{32}$. It is also confirmed that 


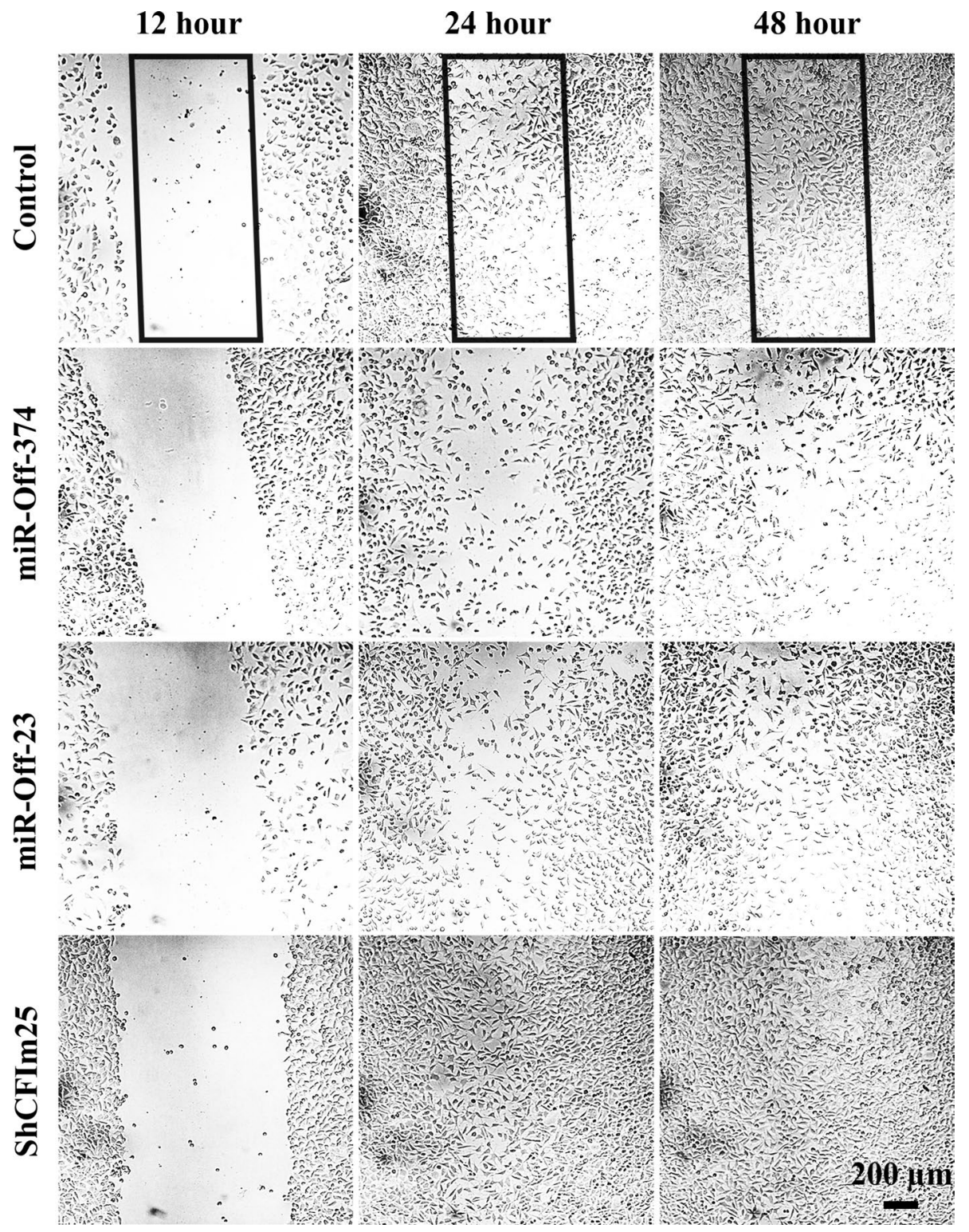

Figure 4. Wound healing assay of transducted MDA-MB-231 cells. Migration of transducted MDA-MB-231 cells toward the scratched area (rectangle) is monitored by 12 -h intervals.

activation of APA sites can affect the migratory capacity of cancer cells ${ }^{33}$ as well as fibroblasts ${ }^{34}$. According to the results of wound healing assay, regulation of CFIm 25 expression by miR-23 and 374 directly controls breast cancer cells' migration and invasion. Since invasion and metastasis are the underlying causes of poor long-term survival of breast cancer patients ${ }^{35}$, regulation of CFIm 25 via miRs could be considered as a promising strategy for inhibition of metastasis in future clinical studies.

Parental genes of mRNAs with shorter 3'-UTRs, are more prone to up-regulation during tumorigenesis due to increased escaping from miRNA repression. It is determined that almost $67 \%$ genes with shorter $3^{\prime}$-UTRs in tumors, have lost at least one predicted miRNA-binding site ${ }^{36}$. In some cases, overexpression of oncogenic proteins comes up without regulatory intervenience of the proto-oncogenes. One example of this phenomenon is cancer cell lines (such as MDA-MB-231) that express substantial amounts of mRNA isoforms with shortened $3^{\prime}$-UTRs ${ }^{30,31}$. CFIm25 is a regulatory protein that controls key factors such as EGFR and MYC that are involved in NF- $\kappa B$ and MAPK/ERK pathways ${ }^{31,37,38}$. Our finding suggests that regulation of proto-oncogenes by CFIm 25 , could be mediated via APA and shortening of 3'UTR of their corresponding mRNAs, which eventually result in progression of breast cancer. The $M Y C, P T E N$ and $A K T 2$ proto-oncogenes are more responsive to CFIm 25 than EGFR and $A K T 3$, since they have several potential interaction sites either at proximal or distal of their mRNA's 3'-UTRs (Table 1). Tuning impact of CFIm 25 on MYC, PTEN and AKT2 will then control downstream phenomena such as glutamine metabolism in cancer cells ${ }^{14}$, which could be applied as an strategy for targeted therapy ${ }^{39}$. Besides, $3^{\prime}$-UTR of EGFR's mRNA contain multiple microRNA target sites, which are associated with cell cycle arrest and cell death. As long as CFIm25 interacts with distal region of EGFR's 3'-UTR mRNA (Table 1), 


\section{$\square 12 \mathrm{~h} \square 24 \mathrm{~h} \square 4 \mathrm{Sh}$}

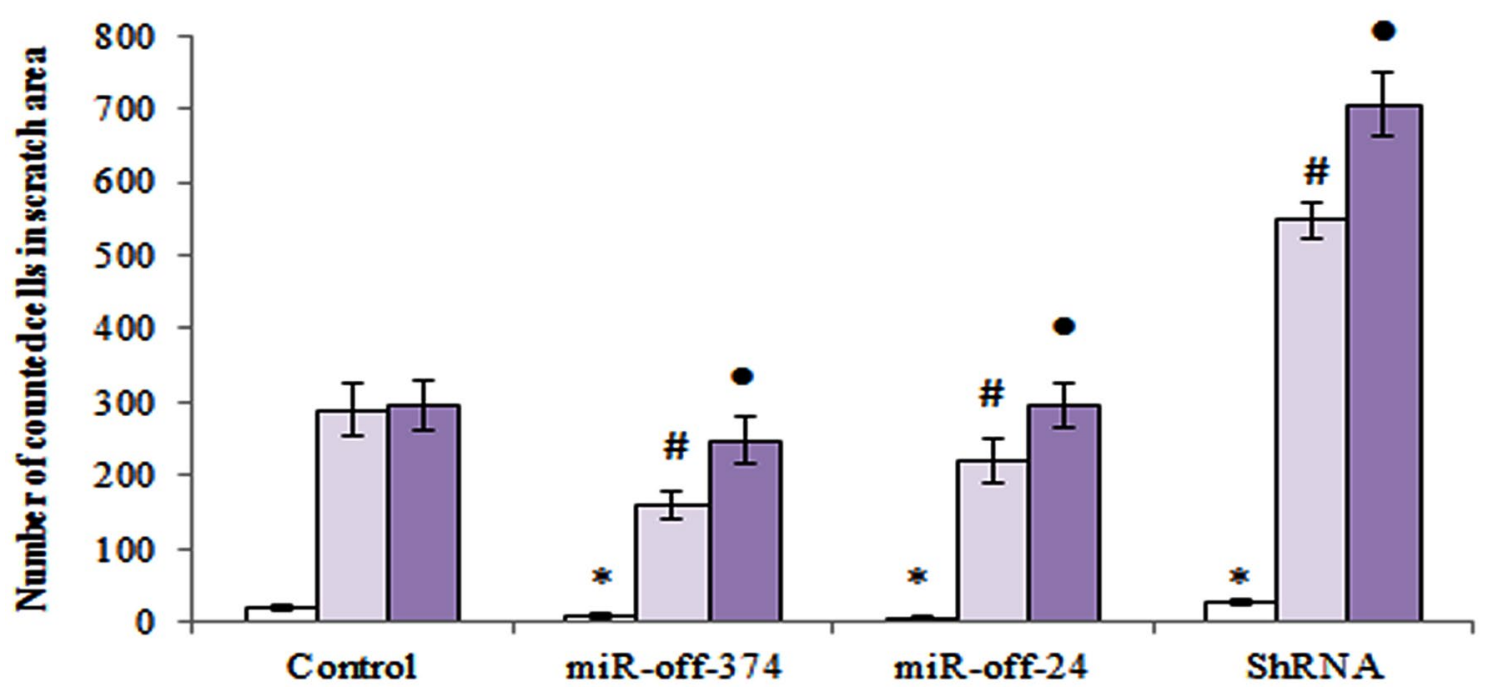

Figure 5. Time course of MDA-MB-231 migration from scratch borders toward the empty area. Diagram of migrated cells to the area of interest that are counted using ImageJ software. Columns are mean of three experiments and error bars represent standard deviation. The (asterisk, hash and filled circle) above the bars, represents the significant difference respect to the control reference at 12, 24 and $48 \mathrm{~h}$, respectively.

suppression of breast cancer tumorigenicity by miRNAs is highly plausible ${ }^{40}$. In a similar way, interaction of CFIm 25 with distal region of $A K T 33^{\prime}$-UTR mRNA, facilitates down-regulation of $A K T 3$, which then regulates migration and metastasis in breast cancer cells ${ }^{41}$. These two examples can clarify how CFIm 25 interaction with distal region of EGFR and AKT3 3'-UTR mRNAs, can facilitates suppression of breast cancer tumorigenicity and metastasis, respectively.

\section{Conclusion}

In summary, CFIm 25 is down-regulated in human breast cancer tissues compared to the adjacent non-cancerous breast tissues. Besides, CFIm 25 mRNA is also regulated by several miRNAs including $m i R-23, m i R-24, m i R-27$, $m i R-135, m i R-182$ and $m i R-374$. Since CFIm 25 is a key factor in cancer proliferation, identification of its modulators, such as miRNAs, facilitates development of molecular-targeted therapeutics for various cancers including breast cancer. Our in-vitro results confirm functionality of certain miRNAs toward regulation of 3'-UTR via CFIm 25 in breast cancer cells, which then introduce it as an emerging field of study in pre-clinical studies of breast cancer.

\section{Methods}

Cell lines and patient sample collection. Hundred fresh frozen samples of human breast cancer tumors and their normal adjacent tissue obtained from the Iran national tumor bank of Cancer Institute (Imam Khomeini hospital, Tehran University of medical sciences, Tehran, Iran). Sample collection performed according to the international tumor bank SOP protocol and written informed consent obtained from patients. This study was approved by the cancer institute of Imam Khomeini Hospital, Tehran, Iran. SOP protocol and consent designed in accordance with ethical standards of Tehran University of medical sciences and with the 1964 Helsinki declaration and its later amendments or comparable ethical standards.

Human embryonic kidney cell line HEK293T (RRID:CVCL_0063) and human breast cancer cell line MDAMB-231 (RRID:CVCL_0062) were obtained from Pasteur Institute of Iran (IPI) and Iranian biological resource center, respectively. Aforementioned institutes check the authenticity of human cell lines via DNA profiling annually. The obtained cell lines cultured in Dulbecco's modified Eagle's Medium (DMEM; Gibco), supplemented with $10 \%$ fetal bovine serum (FBS; Gibco) and $1 \%$ penicillin-streptomycin (Gibco) at $37^{\circ} \mathrm{C}$ with $5 \% \mathrm{CO}_{2}$ incubation.

Bioinformatic analysis. The miRNAs that were susceptible to target CFIm25 mRNA 3'-UTR acquired using prediction web servers such as TargetScan, miRWalk (version 3.0) and DianaTool. The standard cutoff for screening predicted miRNAs was 8 mer, at the most, while the percentage of context ++ score (CS) did not exceed $95 \%$. Predicted miRNAs with the high score were investigated in the miRCancer database. Finally, miRNAs with the oncogenic roles were selected for the rest of the research, including $m i R-23, m i R-24, m i R-27, m i R-96, m i R-$ 135, $m i R-182, m i R-221, m i R-222$ and $m i R-374$.

In order to find out if CFIm 25 efficiently targets $3^{\prime}$-UTR of proto-oncogenes' mRNAs, three steps were followed. First, 3'-UTR mRNA of twenty proto-oncogenes that known to be involved in breast cancer ${ }^{42}$, retrieved from UTR database ${ }^{43}$. Second, twenty retrieved proto-oncogenes including; NM_053056.2, NM_001759.4, NM_000077.4, JX391994.1, S67388.1, NM_005163.2, NM_001626.6, NM_005465.5, BC040540.1, AF067844.1, 
L78833.1, U43746.1, KX710182.1, XM_011512894.2, XM_024450643.1, NM_002467.6, NM_002745.4, NM_005343.4, NM_005228.5 and NM_005417.4, were tested as a matter of proximal or distal placement of CPSF interaction site at polyadenylation signal (A[A/T]TAAA) and their neighbor CFIm 25 interaction site (TGTA). The PAS that is closest to the $5^{\prime}$ end of the $3^{\prime}$-UTR is considered as "proximal" and the other PAS(s) in the downstream of $3^{\prime}$-UTR considered as distal ${ }^{44}$. Third, the retrieved $3^{\prime}$-UTRs beside CFIm25 (O43809) and CPSF1 (Q10570) submitted to the RNA-Protein Interaction Prediction (RPISeq) server to estimate their interaction probabilities ${ }^{45}$. Interaction probabilities ranged from zero to one. Optimal RNA/protein interactions were the ones with interaction probabilities of more than 0.5 , which represented accuracy of the Random Forest (RF) classifiers in cross-validation evaluation experiments on benchmark datasets.

RNA extraction and qRT-PCR. Total RNA extraction of cell line, human breast cancer tissues and normal specimens was performed using Trizol (Invitrogen) in accordance with manufacturer's protocol and previous study $^{22}$. Then, $500 \mathrm{ng}$ of total RNA was reverse transcribed to synthesize cDNA with random hexamer primers and Fermentas kit (for CFIm25 gene) or BON RT adaptor primer and BONmiR kit (for miRNA genes). A total of $1 \mu \mathrm{L}$ of cDNA was amplified using SYBR Green Real-time PCR Master Mix (Takara) and performed on ABI 157 PRISM 7,500 real-time PCR System (Applied Biosystems). The relative expression levels of the CFIm 25 and miRNA genes calculated using the $2^{-\Delta \Delta \mathrm{Ct}}$ method, with normalization against $\beta$-actin and SNORD47 internal controls, respectively. Three biological replicates were performed for each set of experiments. The qRT-PCR primer sequences are listed in supplementary Table 1 and 2.

In clinical samples, the mean of CFIm 25 and $\beta$-actin Cts between all of the normal samples was determined and compared with the obtained Cts of each patient. This procedure adopted from our previous studies with minor modifications ${ }^{20,46}$.

Based on the results, tumors were classified as low and high/equal CFIm25-expressing tumors using the median of the normal samples as a cut-off. Next, the relative gene expression of predicted miRNAs in two groups of patients was calculated by comparing obtained Cts with the mean Cts of normal samples.

Plasmids, viral vectors construction and luciferase assay. For the construction of miRNA-expressing vectors, genomic fragment consists of the stem-loop structure of each miRNA and theirs flanking genomic sequences, cloned into the mammalian expression vector $p L E X$.jred and $p C D H$.TurboGFP at downstream of the cytomegalovirus (CMV) promoter. The PCR primers used for miRNAs cloning were listed in supplementary Table 2. Empty vector without any cloned sequence ( $p L E X-C t r l / p C D H-C t r l)$ was used as control in accordance with Furukawa et al. method ${ }^{47}$.

HEK293T cells were transiently co-transfected by miRNA-expressing lentivectors (or empty backbones), $p P A X 2$ plasmid (packaging plasmid), and $p M D G$ plasmid (containing $v s v-G$ ) using calcium phosphate. Lentivirus supernatants were harvested for two or three times, every $12 \mathrm{~h}$, concentrated by ultracentrifuge at $47,000 \times g$ for $2 \mathrm{~h}$ at $4{ }^{\circ} \mathrm{C}$. Lentivirus titer examined by flow cytometry analysis of reporter positive $293 \mathrm{~T}$ cells (Attune Acoustic Focusing Flow Cytometer, ABI, FlowJo software $)^{48}$.

To down-regulate $m i R-23$ and $m i R-374$ expression, $p$ Lenti-III-miR-Off constructs containing GFP marker purchased from ABM Company and packaged into lentiviruses particles. For luciferase assay, full length of CFIm 25 3'-UTR amplified by PCR using the forward 5'-GCAAGCTTAGCTGTTCTTCTGCC-3' and reverse 5'-GCA CTAGTGTCATCAAAATATTTATTAA-3', respectively. The CFIm25 3'-UTR was then cloned to downstream of the luciferase gene in the $p S I C H E C K 2$ vector (Promega). Mutant stem-loop forms of each miRNA, harboring two mutations in seed sequence, cloned in the lentiviral vector and named Mut-miR, which was considered as negative control. The HEK293 cells were transiently transfected with wild type or mutant miRNA-expressing vector and CFIm25-pSICHECK2 vector using lipofectamine 2000 (Invitrogen) ${ }^{49}$.

Luciferase assay performed after $48 \mathrm{~h}$ of post-transfection, using the dual-luciferase reporter assay system (Promega). Renilla luciferase signal normalized against firefly luciferase activity to monitor transfection efficiency. Data are the means of experiments performed in triplicate.

Western blot. The MDA-MB-231 cells were lysed using cell lysis buffer (50 Mm Tris, $\mathrm{pH}=8.0,150 \mathrm{mM}$ $\mathrm{NaCl}, 1 \% \mathrm{NP}-40,0.5 \%$ sodium deoxycholat, $1 \mathrm{mM}$ sodium fluoride, $1 \mathrm{mM}$ sodium orthovanadate, $1 \mathrm{mM}$ EDTA). The polypeptide component were resolved by electrophoresis at $200 \mathrm{~V}$ using $12 \%$ SDS-polyacrylamide gel electrophoresis (PAGE) transferred into poly vinyl den fluoride membrane and immersed in $5 \%$ non-fat milk powder over one hour at room temperature. Upon completion of the transfer, membranes were incubated with CFIm 25 and $\beta$-actin primary antibodies (Abcam). After washing, membranes probed with horseradish peroxidase-conjugated secondary antibodies $(1: 1,000, \mathrm{Abcam})$ and developed for detection by chemiluminescence on Kodak X-film. In order to quantify bands in western blot gel, acquired images of CFIm 25 and beta actin bands with quality of 75 out of 100 compared as a matter of expression level, using Image J software. Higher number of averaged white pixels considered as higher amount of expression.

Short hairpin RNA (shRNA) design and experiments. For knockdown endogenous CFIm25, target sequence cloned as shRNA format in the $p C D H$-turboGFP. The shCFIm 25 target sequence is $5^{\prime}$-GCCTCA TTCTTATTTCAAGAT-3'. An empty shRNA vector (shCtrl) used as a negative control. The MDA-MB-231cells seeded into a six-well plate and transfected by $p C D H$-shCFIm 25 or $p C D H$-shCtrl and lipofectamine 3,000 (Invitrogen) according to the manufacturer instruction.

Cell viability assay. For investigation of cell proliferation, experiments performed into three groups. In the first group, MDA-MB-231 cells transduced with $m i R-O f f-23 / m i R-O f f-374$ expressing vectors. In the second 
group, MDA-MB-231 cells that were co-transduced with $m i R-O f f-23 / m i R-O f f-374$ expressing vectors were transfected by $p C D H$-shCFIm 25. In the third group, MDA-MB-231 cells transfected merely by $p C D H$-shCFIm 25 . At 48, 72 and $96 \mathrm{~h}$ after transduction/transfection, $10 \mu \mathrm{L}$ of MTT reagent added into wells and incubated for next three hours. Next, $100 \mu \mathrm{L}$ DMSO was added and the resulting absorbance measured at $550 \mathrm{~nm}$ using a multi-well spectrophotometer (Bio-Tek).

Wound healing assay. Wound healing assay performed to evaluate MDA-MB-231 cell migration. The MDA-MB-231 cells seeded in 24-well tissue culture plate until their growth rate reached a confluence of $\sim 80 \%$. The monolayer scratched gently with a $20-\mu \mathrm{L}$ pipette tip across the center of the well. After scratching, wells washed twice with medium to remove the detached cells. Cell migration to the scratch area monitored for additional $48 \mathrm{~h}$ and photographed every $12 \mathrm{~h}$. Number of cells moved from the border of scratch toward the empty area considered as the "migration index". Related calculations performed by ImageJ software.

Received: 21 September 2019; Accepted: 24 June 2020

Published online: 14 July 2020

\section{References}

1. Sotiriou, C. et al. Gene expression profiling in breast cancer: understanding the molecular basis of histologic grade to improve prognosis. J. Natl. Cancer Inst. 98, 262-272 (2006).

2. Ong, C.-T. \& Corces, V. G. Enhancer function: new insights into the regulation of tissue-specific gene expression. Nat. Rev. Genet. 12, 283. https://doi.org/10.1038/nrg2957 (2011).

3. Tian, B. \& Manley, J. L. Alternative polyadenylation of mRNA precursors. Nat. Rev. Mol. Cell Biol. 18, 18 (2017).

4. Tian, B. \& Manley, J. L. Alternative cleavage and polyadenylation: the long and short of it. Trends Biochem. Sci. 38, 312-320 (2013).

5. Ustyantsev, I., Golubchikova, J., Borodulina, O. \& Kramerov, D. Canonical and noncanonical RNA polyadenylation. Mol. Biol. 51, 226-236 (2017).

6. Li, W. et al. Distinct regulation of alternative polyadenylation and gene expression by nuclear poly (A) polymerases. Nucleic Acids Res. 45, 8930-8942 (2017).

7. Colgan, D. F. \& Manley, J. L. Mechanism and regulation of mRNA polyadenylation. Genes Dev. 11, 2755-2766 (1997).

8. Elkon, R., Ugalde, A. P. \& Agami, R. Alternative cleavage and polyadenylation: extent, regulation and function. Nat. Rev. Genet. 14, 496 (2013).

9. Lutz, C. S. \& Moreira, A. Alternative mRNA polyadenylation in eukaryotes: an effective regulator of gene expression. Wiley Interdiscip. Rev. RNA 2, 22-31 (2011).

10. Di Giammartino, D. C., Nishida, K. \& Manley, J. L. Mechanisms and consequences of alternative polyadenylation. Mol. Cell 43, 853-866 (2011).

11. Mayr, C. \& Bartel, D. P. Widespread shortening of 3' UTRs by alternative cleavage and polyadenylation activates oncogenes in cancer cells. Cell 138, 673-684 (2009).

12. Misra, A. \& Green, M. R. From polyadenylation to splicing: Dual role for mRNA 3’end formation factors. RNA Biol. 13, 259-264 (2016).

13. Tan, S. et al. NUDT21 negatively regulates PSMB2 and CXXC5 by alternative polyadenylation and contributes to hepatocellular carcinoma suppression. Oncogene, 37, 4887-4900 (2018).

14. Masamha, C. P. et al. CFIm25 regulates glutaminase alternative terminal exon definition to modulate miR-23 function. RNA 22, 830-838 (2016).

15. Masamha, C. P. et al. CFIm25 links alternative polyadenylation to glioblastoma tumour suppression. Nature 510, 412 (2014).

16. He, L. \& Hannon, G. J. MicroRNAs: small RNAs with a big role in gene regulation. Nat. Rev. Genet. 5, 522 (2004).

17. Cai, Y., Yu, X., Hu, S. \& Yu, J. A brief review on the mechanisms of miRNA regulation. Genom. Proteom. Bioinform. 7, 147-154 (2009).

18. Tasharrofi, N. et al. Survival improvement in human retinal pigment epithelial cells via Fas receptor targeting by miR-374a. J. Cell. Biochem. 118, 4854-4861 (2017).

19. Mobarra, N. et al. Overexpression of microRNA-16 declines cellular growth, proliferation and induces apoptosis in human breast cancer cells. Vitro Cell. Dev. Biol. Anim. 51, 604-611 (2015).

20. Kouhkan, F. et al. MicroRNA-129-1 acts as tumour suppressor and induces cell cycle arrest of GBM cancer cells through targeting IGF2BP3 and MAPK1. J. Med. Genet. 53, 24-33 (2016).

21. Soufi-Zomorrod, M. et al. MicroRNAs modulating angiogenesis: miR-129-1 and miR-133 act as angio-miR in HUVECs. Tumor Biol. 37, 9527-9534 (2016).

22. Pirooz, H. J. et al. Functional SNP in microRNA-491-5p binding site of MMP9 $3^{\prime}-$ UTR affects cancer susceptibility. J. Cell. Biochem. 119, 5126-5134 (2018).

23. Zomorrod, M. S., Kouhkan, F., Soleimani, M., Aliyan, A. \& Tasharrofi, N. Overexpression of miR-133 decrease primary endothelial cells proliferation and migration via FGFR1 targeting. Exp. Cell Res. 369, 11-16 (2018).

24. Peng, Y. \& Croce, C. M. The role of MicroRNAs in human cancer. Signal Transduct. Target. Ther. 1, 15004. https://doi.org/10.1038/ sigtrans.2015.4 (2016).

25. Corsini, L. R. et al. The role of microRNAs in cancer: diagnostic and prognostic biomarkers and targets of therapies. Expert Opin. Therap. Targets 16, S103-S109 (2012).

26. Chen, W. et al. Alternative polyadenylation: methods, findings, and impacts. Genom. Proteom. Bioinform. 15, 287-300 (2017).

27. Lembo, A., Di Cunto, F. \& Provero, P. Shortening of 3' UTRs correlates with poor prognosis in breast and lung cancer. PLoS ONE 7, e31129 (2012).

28. Sandberg, R., Neilson, J. R., Sarma, A., Sharp, P. A. \& Burge, C. B. Proliferating cells express mRNAs with shortened 3' untranslated regions and fewer microRNA target sites. Science 320, 1643-1647 (2008).

29. Fu, Y. et al. Differential genome-wide profiling of tandem 3' UTRs among human breast cancer and normal cells by high-throughput sequencing. Genome Res. 21, 741-747 (2011).

30. Wang, Q. et al. Cell cycle regulation by alternative polyadenylation of CCND1. Sci. Rep. 8, 6824 (2018).

31. Lou, J-C. et al. Silencing NUDT21 attenuates the mesenchymal identity of glioblastoma cells via the NF-кB pathway. Front. Mol. Neurosci. 10, 420 (2017).

32. Wang, Y. et al. CFIm 25 inhibits hepatocellular carcinoma metastasis by suppressing the p38 and JNK/c-Jun signaling pathways. Oncotarget 9, 11783 (2018). 
33. Zhu, Z. J. et al. MicroRNA-181a promotes proliferation and inhibits apoptosis by suppressing CFIm25 in osteosarcoma. Mol. Med. Rep. 14, 4271-4278 (2016).

34. Mitra, M. et al. Alternative polyadenylation factors link cell cycle to migration. Genome Biol. 19, 176 (2018).

35. Heimann, R., Lan, F., McBride, R. \& Hellman, S. Separating favorable from unfavorable prognostic markers in breast cancer: the role of E-cadherin. Can. Res. 60, 298-304 (2000).

36. Xia, Z. et al. Dynamic analyses of alternative polyadenylation from RNA-seq reveal a 3'-UTR landscape across seven tumour types. Nat. Commun. 5, 5274 (2014).

37. Abadi, M. H. J. N. et al. CFIm25 and alternative polyadenylation: conflicting roles in cancer. Cancer Lett. 459, 112-121 (2019).

38. Zhang, L. \& Zhang, W. Knockdown of NUDT21 inhibits proliferation and promotes apoptosis of human K562 leukemia cells through ERK pathway. Cancer Manag. Res. 10, 4311 (2018).

39. Hoerner, C. R., Chen, V. J. \& Fan, A. C. The 'Achilles Heelof metabolism in renal cell carcinoma: glutaminase inhibition as a rational treatment strategy. Kidney Cancer, 1-17 (2019).

40. Webster, R. J. et al. Regulation of epidermal growth factor receptor signaling in human cancer cells by microRNA-7. J. Biol. Chem. 284, 5731-5741. https://doi.org/10.1074/jbc.M804280200 (2009).

41. Grottke, A. et al. Downregulation of AKT3 increases migration and metastasis in triple negative breast cancer cells by upregulating S100A4. PLoS ONE 11, e0146370. https://doi.org/10.1371/journal.pone.0146370 (2016).

42. Lee, E. Y. \& Muller, W. J. Oncogenes and tumor suppressor genes. Cold Spring Harbor Perspect. Biol. 2, a003236 (2010).

43. Grillo, G. et al. UTRdb and UTRsite (RELEASE 2010): a collection of sequences and regulatory motifs of the untranslated regions of eukaryotic mRNAs. Nucleic Acids Res. 38, D75-D80 (2009).

44. Zhu, Y. et al. Molecular mechanisms for CFIm-mediated regulation of mRNA alternative polyadenylation. Mol. Cell 69, 62-74 (2018).

45. Muppirala, U. K., Honavar, V. G. \& Dobbs, D. Predicting RNA-protein interactions using only sequence information. BMC Bioinform. 12, 489 (2011).

46. Torabi, S. et al. miR-455-5p downregulation promotes inflammation pathways in the relapse phase of relapsing-remitting multiple sclerosis disease. Immunogenetics 71, 87-95 (2019).

47. Furukawa, N. et al. Optimization of a microRNA expression vector for function analysis of microRNA. J. Control. Release 150, 94-101 (2011).

48. Mohammadi, Z. et al. A lentiviral vector expressing desired gene only in transduced cells: an approach for suicide gene therapy. Mol. Biotechnol. 57, 793-800 (2015).

49. Parsons, C. J. et al. Mutation of the 5'-untranslated region stem-loop structure inhibits al (I) collagen expression in vivo. J. Biol. Chem. 286, 8609-8619 (2011).

\section{Author contributions}

F.K. and S.M.A.H. designed the study, M.T., G.S., performed the experiments. I.R. analyzed the data, provided intellectual support and expertise toward interpretation of results. À.E. collaborated in cell line preparation and culture set up. All authors contributed to the edition and the critical review of the manuscript.

\section{Competing interests}

The authors declare no competing interests.

\section{Additional information}

Supplementary information is available for this paper at https://doi.org/10.1038/s41598-020-68406-3.

Correspondence and requests for materials should be addressed to F.K.

Reprints and permissions information is available at www.nature.com/reprints.

Publisher's note Springer Nature remains neutral with regard to jurisdictional claims in published maps and institutional affiliations.

Open Access This article is licensed under a Creative Commons Attribution 4.0 International License, which permits use, sharing, adaptation, distribution and reproduction in any medium or format, as long as you give appropriate credit to the original author(s) and the source, provide a link to the Creative Commons license, and indicate if changes were made. The images or other third party material in this article are included in the article's Creative Commons license, unless indicated otherwise in a credit line to the material. If material is not included in the article's Creative Commons license and your intended use is not permitted by statutory regulation or exceeds the permitted use, you will need to obtain permission directly from the copyright holder. To view a copy of this license, visit http://creativecommons.org/licenses/by/4.0/.

(C) The Author(s) 2020 